\title{
Heparin Cryoprecipitation Reduces Plasma Levels of Non-Traditional Risk Factors for Atherosclerosis in vitro
}

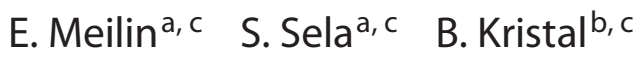 \\ aEliachar Research Laboratory, ${ }^{\text {b }}$ Department of Nephrology, Western Galilee Hospital, Nahariya, and \\ 'Bruce Rappaport Faculty of Medicine, Technion, Haifa, Israel
}

\section{Key Words}

Atherosclerosis $\cdot$ Non-traditional risk factors $\cdot$ Oxidized molecules - Inflammation - Heparin cryoprecipitation of plasma

\begin{abstract}
Aims: To show that heparin cryoprecipitation (HCP), an in vitro method of plasma purification, reduces the levels of in vivo modified proteins and non-traditional risk factors from plasma of atherosclerotic hemodialysis (HD) patients. Methods: $\mathrm{HCP}$ was applied to plasma obtained from HD patients and controls, forming a precipitate - cryogel. Levels of fibrinogen, albumin, CRP, TNF- $\alpha$, IL- 6 , advanced oxidation protein products, carbonylated fibrinogen and carbonylated albumin were determined in plasma before and after applying HCP and in the cryogel. Results: Treatment of HD plasma with $\mathrm{HCP}$, beyond the significant reduction of the increased levels of all the above-mentioned molecules, reduced fibrinogen, TNF- $\alpha$, carbonylated fibrinogen and carbonylated albumin to control levels which were simultaneously found in the cryogel. Conclusions: HCP applied to plasma enables the simultaneous precipitation of modified molecules and circulating non-traditional risk factors for atherosclerosis. This study may serve as a base for the future development of a clinical purification technique.
\end{abstract}

Copyright $\odot 2008$ S. Karger AG, Basel
Atherosclerosis and cardiovascular diseases (CVD), which are associated with inflammation, oxidative stress and endothelial dysfunction, remain the major causes of death in the modern world. Although major progress has been made in understanding and modulating risk factors for atherosclerosis, it is still the primary cause of morbidity and mortality in patients suffering from end-stage renal failure treated with chronic hemodialysis (HD) [1]. Most atherosclerotic patients have multiple cardiovascular risk factors, traditional and non-traditional, which potentiate each other, and are usually treated by the pharmacological approach. The classical, traditional, risk factors are usually disease-orientated like hypertension, dyslipidemia and diabetes mellitus, while the non-traditional risk factors focus on specific molecules usually associated with oxidative stress and inflammation processes [1-3].

In inflammation, blood levels of acute phase proteins and cytokines such as fibrinogen, albumin, C-reactive protein (CRP), tumor necrosis factor- $\alpha$ (TNF- $\alpha$ ) and interleukin (IL)-6 are modulated, while in severe oxidative stress molecular modifications such as the addition of carbonyls on plasma proteins occur $[1,4,5]$. Albumin and fibrinogen are major plasma proteins which get modified by oxidative stress. Beyond being irreversible and irreparable, carbonylization of proteins by oxidation [6] can lead to diverse functional consequences: tendency of the

\section{KARGER \\ Fax +41613061234 E-Mail karger@karger.ch} www.karger.com
Edna Meilin

Eliachar Research Laboratory, Western Galilee Hospital

Naharyia 221000 (Israel)

Tel. +972 4910 7769, Fax +97249107469

E-Mail ednameilin@yahoo.com 
protein to aggregate, to be less soluble and to precipitate $[5,7,8]$. In addition, oxidation of albumin, for example, will reduce drug-binding properties and antioxidant properties and may induce tissue factor activity in culture of human umbilical vein endothelial cells [9]. Oxidized albumin is able to trigger the oxidative burst of human neutrophils $[10,11]$ while oxidized fibrinogen loses the ability to form a solid clot $[5,12]$, may induce platelet aggregation in the circulation, and enhance IL- 8 secretion from human umbilical vein endothelial cells [13].

This study aimed to specifically reduce the levels of modified molecules, especially those that could serve as non-traditional risk factors for atherosclerosis. This was achieved by using the newly developed heparin cryoprecipitation (HCP) method, which is based on an interaction between heparin and proteins, combining freezing temperatures and centrifugation. This precipitation of modified molecules and risk factors forms a pellet, called cryogel. The interaction between heparin and fibrinogen in cooling temperatures has previously been described by Smith et al. [14].

The different effects of temperature, centrifugation and heparin, comprising the HCP method, were evaluated on in vitro modified/untreated fibrinogen and albumin. The HCP method was also applied to commercial TNF- $\alpha$. Plasma before and after HCP, and cryogels were obtained from atherosclerotic HD patients and the levels of various inflammatory mediators were compared to healthy controls.

HCP preferentially reduces the levels of oxidized proteins, and almost no native proteins, from plasma of atherosclerotic patients. Simultaneously, precipitation of non-modified circulating risk factors was observed from atherosclerotic plasma.

\section{Material and Methods}

\section{Blood Samples}

Blood was drawn from 25 patients (age range 48-73) with endstage renal disease on chronic HD treatment, three times a week for $4 \mathrm{~h}$. All the included patients were clinically diagnosed as suffering from accelerated atherosclerosis. 20 healthy controls (HC), age- and gender-matched, served as the control group. Blood from HD patients was always drawn before the start of a dialysis session. The inclusion of HC subjects in the study was based upon clinical examination with laboratory confirmation. Informed consent was obtained from all the patients and subjects participating in this study according to the protocol approved by the institutional committee in accordance with the Helsinki Declaration.
In vitro Oxidation of Fibrinogen and Albumin

Fibrinogen and albumin (Sigma-Aldrich, St. Louis, Mo., USA) were oxidized in vitro using the metal-catalyzed oxidation system comprised of iron and ascorbate to yield a highly oxidized (carbonylated) protein, according to Michellis et al. [15]. Briefly, fibrinogen oxidation $(3.5-6 \mathrm{mg} / \mathrm{ml})$ was carried out for $5 \mathrm{~h}$ at $37^{\circ} \mathrm{C}$ in PBS containing $5 \mathrm{~mm}$ ascorbate (Sigma, St. Louis, Mo., USA) and $100 \mu \mathrm{M} \mathrm{FeCl}$ (Merck, Germany), while albumin oxidation $(28-42 \mathrm{mg} / \mathrm{ml})$ was carried out for $52 \mathrm{~h}$ at $37^{\circ} \mathrm{C}$ in PBS containing $25 \mathrm{mM}$ ascorbate and $100 \mu \mathrm{M} \mathrm{FeCl}_{3}$. The reactions were stopped at $4^{\circ} \mathrm{C}$ by the addition of ethylenediaminetetraacetic acid (EDTA) at $\mathrm{pH} 8.0$ (Sigma) to a final concentration of $1 \mathrm{~mm}$. The oxidizing reagents were removed by overnight dialysis, at room temperature, against PBS.

\section{Determination of Carbonyls Content on Proteins}

Levels of carbonylated fibrinogen and albumin were determined, according to Michelis et al. [15]. Briefly, samples were derivatized with 2,4-dinitrophenylhydrazine and separated on sodium dodecyl sulfate-polyacrylamide gel electrophoresis gels (SDS-PAGE): $6 \%$ for fibrinogen and 10\% for albumin. A fixed amount of the in vitro oxidized fibrinogen/oxidized albumin served as a standard [15]. For each protein studied, two gels were run in parallel, one gel was stained for proteins by Coomassie blue, and the other was transferred on to nitrocellulose membrane in transfer buffer (25 mmol/l Tris, $192 \mathrm{mmol} / \mathrm{l}$ glycine) for Western blot analysis. The detection of carbonyls was performed with rabbit polyclonal anti-dinitrophenyl serum (Sigma) followed by secondary goat anti-rabbit IgG peroxidase conjugate (Sigma). In order to confirm that the carbonyls detected were on fibrinogen/albumin, several nitrocellulose membranes were stripped in water for $30 \mathrm{~min}$ and used again for Western blot analysis of fibrinogen, using rabbit polyclonal anti-fibrinogen serum (ICN Pharmaceuticals, Aurora, Ohio, USA) or albumin using rabbit polyclonal anti-human albumin (Sigma, Rehovot, Israel), followed by secondary goat anti-rabbit IgG peroxidase conjugate (Sigma). All antibodies signals were detected on X-ray films using the chemiluminescence reagents of the EZ-ECL kit (Biological Industries, Beit-Haemek, Israel).

The band densities of the Western blot films and of the Coomassie blue stained gels were analyzed with the BioCapt and BioProfil (Bio-1D) software. The content of carbonyls per fibrinogen or albumin was calculated in each sample relative to the standard oxidized fibrinogen or oxidized albumin [15].

\section{Heparin Cryoprecipitation Method}

Evaluation of Temperature, Centrifugation and Heparin

Effect on Protein Precipitation

The separate effects of the heparin, temperature and centrifugal force, comprising the HCP method, were applied to in vitro oxidized fibrinogen and albumin and to untreated proteins (tables 1, 2).

Solutions of fibrinogen/albumin (Sigma-Aldrich) and their oxidized forms were divided into four tubes after measuring the protein concentration by absorbance at $280 \mathrm{~nm}$. Two tubes contained heparin (14 IU/ml) and two without heparin served as control for each protein (evaluation of the effect of heparin). The concentrations of in vitro fibrinogen/albumin and in vitro oxidized fibrinogen/albumin were measured and compared to their initial concentrations after: a) freezing $\left(-20^{\circ} \mathrm{C}\right.$ for $\left.24 \mathrm{~h}\right)$ and thawing 
Table 1. Effect of heparin, temperature and centrifugation on the precipitation of untreated fibrinogen (UT-FIB) and oxidized fibrinogen (FIB-CO)

\begin{tabular}{lllll}
\hline & $\begin{array}{l}\text { Relative initial } \\
\text { concentration } \\
\text { of fibrinogen }\end{array}$ & $\begin{array}{l}\text { Effect of freezing and } \\
\text { thawing }\left(22^{\circ} \mathrm{C}\right) \text { on } \\
\text { fibrinogen precipitation }\end{array}$ & $\begin{array}{l}\text { Effect of centrifuga- } \\
\text { tion }\left(4^{\circ} \mathrm{C}\right) \text { on fibrino- } \\
\text { gen precipitation }\end{array}$ & $\begin{array}{l}\text { Total precipi- } \\
\text { tation of } \\
\text { fibrinogen }\end{array}$ \\
\hline UT-FIB & $100 \%$ & $-4 \pm 2 \%$ & $-1 \pm 4 \%$ & $-5 \pm 4 \%$ \\
UT-FIB + heparin & $100 \%$ & $-4 \pm 3 \%$ & $-11 \pm 4 \%$ & $-14 \pm 3 \%$ \\
FIB-CO & $100 \%$ & $-21 \pm 6 \%$ & $-25 \pm 10 \%$ & $-40 \pm 10 \%$ \\
FIB-CO + heparin & $100 \%$ & $-19 \pm 7 \%$ & $-37 \pm 17 \%$ & $-49 \pm 16 \%$ \\
\hline
\end{tabular}

Table 2. Effect of heparin, temperature and centrifugation on the precipitation of untreated albumin (UT-ALB) and oxidized albumin (ALB-CO)

\begin{tabular}{llllc}
\hline & $\begin{array}{l}\text { Relative initial } \\
\text { concentration } \\
\text { of albumin }\end{array}$ & $\begin{array}{l}\text { Effect of freezing and } \\
\text { thawing }\left(22^{\circ} \mathrm{C}\right) \text { on } \\
\text { albumin precipitation }\end{array}$ & $\begin{array}{l}\text { Effect of centrifuga- } \\
\text { tion }\left(4^{\circ} \mathrm{C}\right) \text { on albu- } \\
\text { min precipitation }\end{array}$ & $\begin{array}{l}\text { Total precipi- } \\
\text { tation of } \\
\text { albumin }\end{array}$ \\
\hline UT-ALB & $100 \%$ & $-5 \pm 5 \%$ & $-1 \pm 1 \%$ & $-6 \pm 7 \%$ \\
UT-ALB + heparin & $100 \%$ & $-7 \pm 6 \%$ & $0 \pm 3 \%$ & $-7 \pm 6 \%$ \\
ALB-CO & $100 \%$ & $-14 \pm 15 \%$ & $-3 \pm 9 \%$ & $-17 \pm 11 \%$ \\
ALB-CO+ heparin & $100 \%$ & $-11 \pm 14 \%$ & $-14 \pm 7 \%$ & $-23 \pm 13 \%$ \\
\hline
\end{tabular}

$\left(22^{\circ} \mathrm{C}\right)$, b) freezing $\left(-20^{\circ} \mathrm{C}\right.$ for $\left.24 \mathrm{~h}\right)$, thawing $\left(4^{\circ} \mathrm{C}\right)$ and centrifugation at $4^{\circ} \mathrm{C}$ for the evaluation of the combined effect of heparin and centifugation, c) combined effect of heparin, freezing, thawing and centrifugation. The results are shown in tables 1 and 2 . Total precipitation refers to the percentage of precipitation following heparin $(14 \mathrm{IU} / \mathrm{ml})$, freezing, thawing and centrifugation $(800 \mathrm{~g})$.

To summarize the HCP method: the samples were drawn in the presence of $14 \mathrm{IU} / \mathrm{ml}$ of sodium heparin, frozen $\left(-20^{\circ} \mathrm{C}\right.$ for $24 \mathrm{~h})$ and thawed $\left(4^{\circ} \mathrm{C}\right)$, followed by centrifugation $\left(4^{\circ} \mathrm{C}, 800 \mathrm{~g}\right)$ and the immediate removal of the supernatant.

In all studies referring to the use of HCP, the same conditions were applied to all samples.

\section{Effect of HCP on Commercial TNF- $\alpha$}

Purified TNF- $\alpha$ (Sigma-Aldrich) was dissolved in PBS and used for in vitro studies. The levels of purified TNF- $\alpha$ were determined by enzyme-linked immunosorbent assay (Bender MedSystems $\mathrm{GmbH}$, Vienna, Austria) before and after application of the HCP method. Commercial TNF- $\alpha$ was prepared in tubes containing 14 $\mathrm{IU} / \mathrm{ml}$ of sodium heparin (Vacuette ${ }^{\circledR}$, Greiner Bio-One $\mathrm{GmbH}$, Kremsmünster, Austria) (Before HCP), frozen for $24 \mathrm{~h}\left(-20^{\circ} \mathrm{C}\right)$, then thawed to $4^{\circ} \mathrm{C}$ and centrifuged again $(800 \mathrm{~g})$ at $4^{\circ} \mathrm{C}$, followed by the immediate removal of the supernatant (After HCP).

\section{Application of HCP to Plasma}

Blood was drawn into tubes containing $14 \mathrm{IU} / \mathrm{ml}$ of sodium heparin (Vacuette ${ }^{\circledR}$, Greiner Bio-One $\mathrm{GmbH}$ ), centrifuged at $800 \mathrm{~g}$ to obtain cell-free plasma (Plasma before). This plasma was frozen for $24 \mathrm{~h}$, then thawed at $4^{\circ} \mathrm{C}$ and centrifuged again $(800 \mathrm{~g})$ at $4{ }^{\circ} \mathrm{C}$, followed by the immediate removal of the supernatant (Plasma after). This procedure resulted in a pellet referred by us as cryogel. The cryogel was insoluble in cold temperatures but mostly soluble at $37^{\circ} \mathrm{C}$.

Determination of Biochemical and Inflammatory Parameters

Fibrinogen and albumin levels in plasma and cryogel of HD patients and $\mathrm{HC}$ subjects were determined using the Cobas Mira analyzer (Roche Diagnostics, Basel, Switzerland) using the K-assay kit (Kamiya Biomedical Co., Seattle, Wash., USA) for fibrinogen and using a colorimetric assay for albumin (Sentinal Diagnostics, Milan, Italy). Plasma and cryogel levels of IL-6, CRP and TNF- $\alpha$ in HD patients and HC were determined using enzymelinked immunosorbent assay by Bender MedSystems $\mathrm{GmbH}$.

\section{Determination of Advanced Oxidation Protein Products}

Advanced oxidation protein products (AOPP) serve as general markers for the evaluation of protein oxidation. AOPP levels in plasma and cryogel of HD patients and HC were evaluated. Determination of AOPP is based on spectophotometric detection [16]. Plasma samples were diluted 1:5 in PBS; pure acetic acid (99\%; Sigma) was added to all tubes and the absorbance at $340 \mathrm{~nm}$ was measured immediately. A standard calibration curve was performed using Chloramine-T solution (Sigma, The Netherlands), $0-100 \mu \mathrm{mol} / \mathrm{l}$, followed by the addition of $1.16 \mathrm{~mol} / \mathrm{l} \mathrm{po}-$ tassium iodide (Sigma). PBS served as a blank.

Protein Phenotyping of Cryogel

Analysis of the protein contents of the cryogel by sequencing and identification was carried out by mass spectrometry, in the 


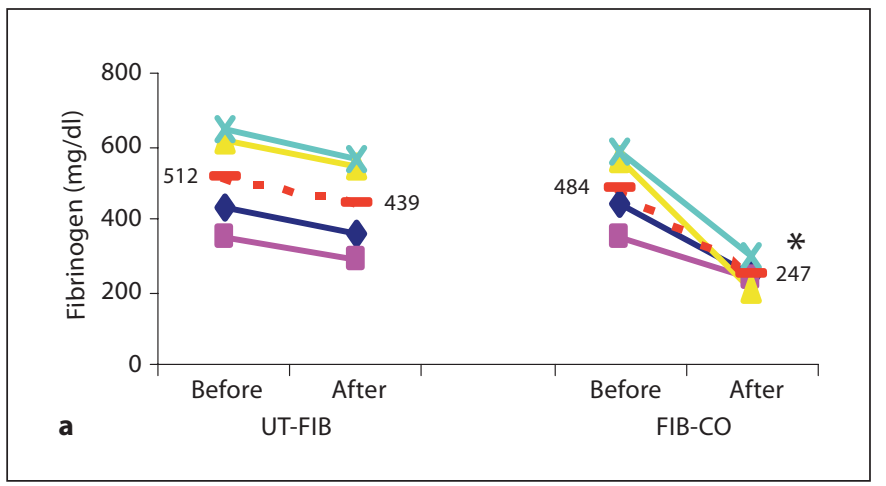

Fig. 1. Effect of HCP on fibrinogen and on in vitro oxidized fibrinogen. a Purified fibrinogen was prepared and divided into two samples: one served as control (untreated; UT-FIB) and the other was oxidized in vitro (FIB-CO). The fibrinogen levels in the two samples were measured before and after the application of HCP method by the Cobas Mira analyzer. The dashed line represents the average results $(n=4) .{ }^{*} \mathrm{p}<0.05$ before vs. after for both

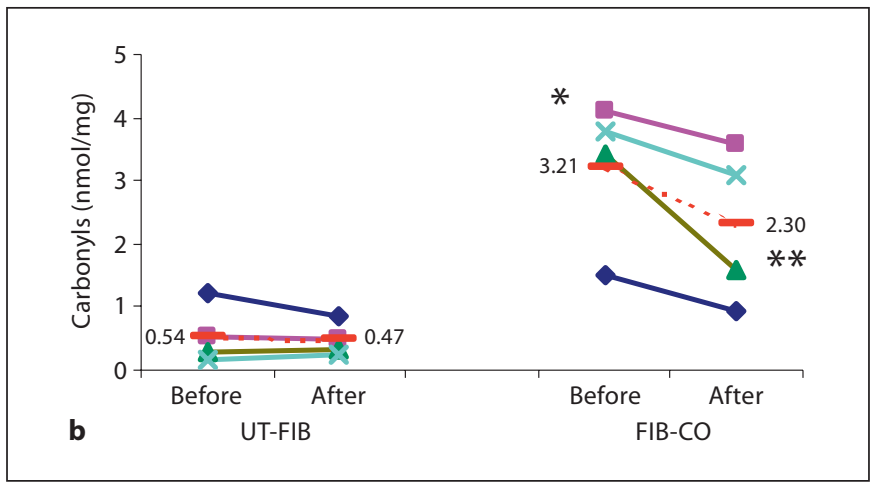

FIB-CO and UT-FIB. $\mathbf{b}$ The effect of HCP on precipitation of carbonylated fibrinogen. The levels of carbonyls on untreated purified fibrinogen and on in vitro oxidized fibrinogen were evaluated by SDS-PAGE before and after application of the HCP method. The dashed line represents the average results $(n=4) .{ }^{*} \mathrm{p}<$ 0.05 FIB-CO before vs. UT-FIB before; ${ }^{* *} \mathrm{p}<0.05$ before vs. after.

protein, which was augmented by the addition of heparin ( 49 vs. $40 \%$ ). Both FIB-CO and UT-FIB precipitated more in the presence of heparin, although modified fibrinogen precipitated more than the native protein (table $1, \mathrm{n}=4)$.

The conditions (heparin $14 \mathrm{IU}$, freezing $-20^{\circ} \mathrm{C}$, thawing $4^{\circ} \mathrm{C}$, centrifugation $800 \mathrm{~g}, 4^{\circ} \mathrm{C}$ ) where we succeeded in precipitating maximum FIB-CO were used in all the following experiments and are referred to hereafter as HCP. The effect of HCP on the precipitation of commercial fibrinogen is also shown in figure 1a.

The in vitro FIB-CO was precipitated from an average of $484 \pm 98 \mathrm{mg} / \mathrm{dl}$ before application of HCP to $247 \pm$ $32 \mathrm{mg} / \mathrm{dl}$ after the application of HCP (49\% decrease), while UT-FIB was precipitated from $512 \pm 127$ to $439 \pm$ $93 \mathrm{mg} / \mathrm{dl}$ (14\% decrease) (fig. 1a).

The levels of carbonyls in the UT-FIB and in vitro FIB$\mathrm{CO}$ are depicted in figure $1 \mathrm{~b}$. Higher levels of carbonyls per fibrinogen molecule were expressed on in vitro FIBCO compared to UT-FIB, $3.21 \pm 1.45$ vs. $0.54 \pm 0.47$ $\mathrm{nmol}$ carbonyl/mg fibrinogen, respectively. HCP significantly reduced the levels of the highly carbonylated fibrinogen molecules, from $3.21 \pm 1.45$ to $2.30 \pm 1.26$ $\mathrm{nmol}$ carbonyl $/ \mathrm{mg}$ (28\% reduction) while in UT-FIB the lower carbonyl levels were non-significantly reduced from carbonyl levels of $0.54 \pm 0.47$ to $0.47 \pm 0.28 \mathrm{nmol}$ carbonyl/mg; the higher the carbonylation, the greater the precipitation. 


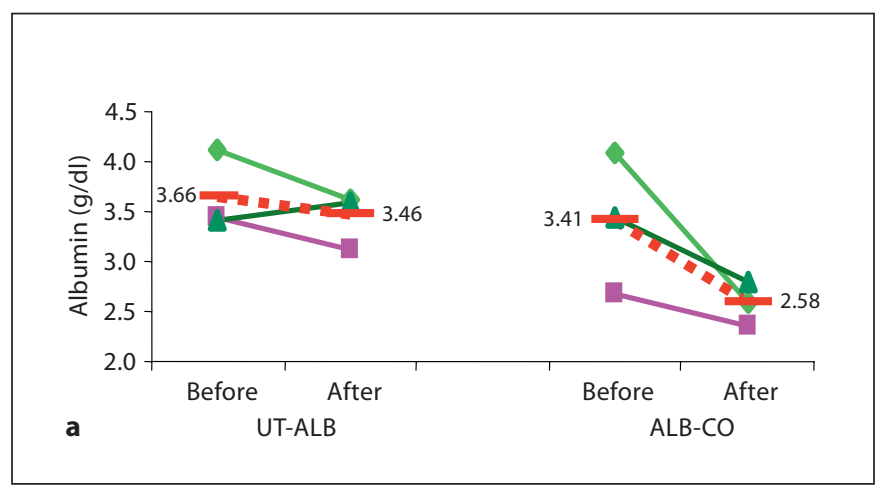

Fig. 2. Effect of HCP on albumin and on in vitro oxidized albumin. a Purified albumin was prepared and divided into two samples: one serves as control (untreated; UT-ALB) and the other was oxidized in vitro (ALB-CO). The albumin levels in the two samples were measured before and after application of the HCP method by the Cobas Mira analyzer. The dashed line represents the average results $(n=3)$. $\mathbf{b}$ Effect of HCP on precipitation of carbon-

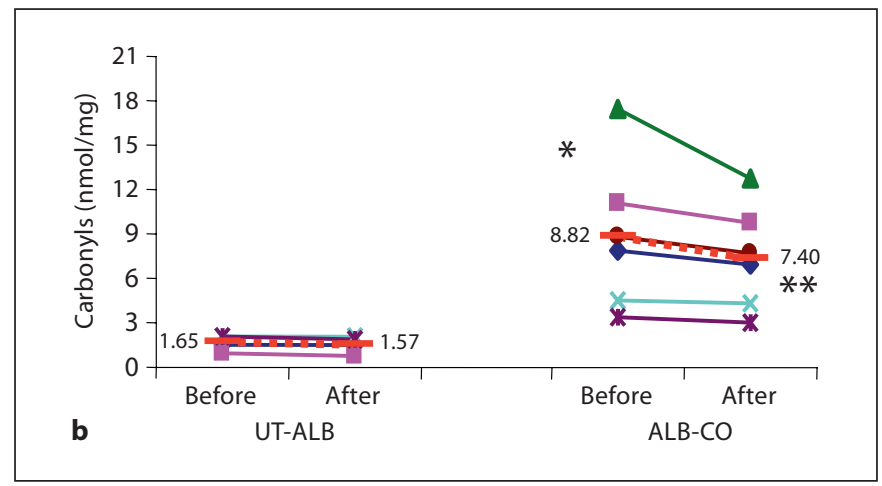

ylated albumin. The levels of carbonyls on untreated purified albumin and on in vitro oxidized albumin were evaluated by SDSPAGE before and after application of the HCP method ( $n=4$ for UT-ALB, $n=6$ for ALB-CO). The dashed line represents the average results. ${ }^{*} \mathrm{p}<0.05$ ALB-CO before vs. UT-ALB before; ${ }^{* *} \mathrm{p}<$ 0.05 before vs. after.

Table 3. Effect of HCP on levels of inflammatory mediators and modified molecules in HD and HC plasma

\begin{tabular}{|c|c|c|c|c|c|c|}
\hline & \multicolumn{3}{|l|}{ HD plasma } & \multicolumn{3}{|l|}{ HC plasma } \\
\hline & before & after & $\Delta$ & before & after & $\Delta$ \\
\hline Fibrinogen, mg/dl & $664 \pm 162 *$ & $388 \pm 140^{* *}$ & $-42 \%$ & $444 \pm 78$ & $283 \pm 142^{* *}$ & $-36 \%$ \\
\hline Albumin, g/dl & $4.02 \pm 0.35^{*}$ & $3.82 \pm 0.29^{* *}$ & $-5 \%$ & $4.64 \pm 0.26$ & $4.61 \pm 0.28$ & \\
\hline $\mathrm{CRP}, \mathrm{mg} / \mathrm{l}$ & $28.1 \pm 2.1^{*}$ & $23.8 \pm 2.0^{* *}$ & $-16 \%$ & $1.3 \pm 0.12$ & $1.2 \pm 0.10$ & $-8 \%$ \\
\hline TNF- $\alpha, \mathrm{pg} / \mathrm{ml}$ & $2.70 \pm 1.29^{*}$ & $0.58 \pm 0.8^{* *}$ & $-78 \%$ & $0.44 \pm 0.90$ & $0.34 \pm 0.67$ & $-24 \%$ \\
\hline IL-6, pg/ml & $5.8 \pm 3.05^{*}$ & $5.13 \pm 2.94^{* *}$ & $-12 \%$ & $0.88 \pm 0.38$ & $0.87 \pm 0.37$ & \\
\hline AOPP, $\mu \mathrm{M}$ & $186 \pm 63^{*}$ & $157 \pm 62^{* *}$ & $-16 \%$ & $69 \pm 6.3$ & $63 \pm 4.8$ & $-12 \%$ \\
\hline FIB-CO, nmol Carb/mg protein & $1.22 \pm 0.58^{*}$ & $0.68 \pm 0.46^{* *}$ & $-44 \%$ & $0.35 \pm 0.15$ & $0.36 \pm 0.21$ & \\
\hline ALB-CO, nmol Carb/mg protein & $8.28 \pm 3.01^{*}$ & $6.85 \pm 2.77^{* *}$ & $-17 \%$ & $3.38 \pm 1.66$ & $2.91 \pm 1.42$ & $-14 \%$ \\
\hline
\end{tabular}

Results are mean \pm SD of the following sample size: fibrinogen, $n=25$ for HD, $n=20$ for HC; albumin and AOPP, $n=20$ for HD, $\mathrm{n}=10$ for HC; CRP, TNF- $\alpha$, and IL-6, $\mathrm{n}=8$ for HD, $\mathrm{n}=4$ for HC; FIB-CO and ALB-CO, $\mathrm{n}=10$ for HD, $\mathrm{n}=5$ for HC. Before and after relate to the application of the HCP procedure. FIB-CO = Carbonylated fibrinogen; ALB-CO = carbonylated albumin.

${ }^{*} \mathrm{p}<0.05 \mathrm{HD}$ before vs. HC before; ${ }^{* *} \mathrm{p}<0.05$ before HCP vs. after HCP.

Commercial untreated albumin (UT-ALB), with or without heparin, followed by freezing and thawing $\left(22^{\circ} \mathrm{C}\right)$, resulted in the precipitation of a small percentage of albumin (about 5-7\%), indicating that heparin, freezing and thawing had very little effect on the precipitation of native UT-ALB. Cold temperatures and centrifugation of these samples did not add to the precipitation of native albumin (table 2, $\mathrm{n}=3$ ).

Freezing and thawing of the in vitro oxidized albumin (ALB-CO), with and without heparin, resulted in about
11-14\% precipitation of the protein. The addition of cold temperatures and centrifugation resulted in an additional precipitation of the protein, which was augmented by the presence of heparin (23 vs. $17 \%$ ). ALB-CO precipitated to a greater extent in the presence of heparin (table 2).

The particular effect of HCP on the precipitation of commercial albumin is also shown in figure $2 \mathrm{a}$.

ALB-CO was precipitated from $3.41 \pm 0.7$ to $2.58 \pm$ $0.3 \mathrm{~g} / \mathrm{dl}$ (23\% decrease) while UT-ALB was precipitated 
from $3.66 \pm 0.5$ to $3.46 \pm 0.3 \mathrm{~g} / \mathrm{dl}$ (7\% decrease) (fig. 2a).

The levels of carbonyls on untreated and oxidized albumin are depicted in figure $2 \mathrm{~b}$. ALB-CO expressed higher levels of carbonyls per albumin molecule compared to UT-ALB and had a higher carbonyl content of $8.82 \pm 5.05$ vs. $1.65 \pm 0.46 \mathrm{nmol}$ carbonyl $/ \mathrm{mg}$ albumin, respectively. HCP reduced significantly highly carbonylated albumin molecules from $8.82 \pm 5.05$ to $7.40 \pm 3.54$ $\mathrm{nmol}$ carbonyl $/ \mathrm{mg}$ ( $16 \%$ decrease) albumin while in UTALB the carbonyl levels were not reduced $(1.65 \pm 0.46$ to $1.57 \pm 0.62 \mathrm{nmol}$ carbonyl $/ \mathrm{mg}$ albumin) following HCP; the higher the carbonylation, the greater precipitation.

The difference of the carbonyl content on in vitro FIB$\mathrm{CO}$ vs. ALB-CO $(8.82 \pm 5.05 \mathrm{nmol}$ carbonyl $/ \mathrm{mg}$ for albumin vs. $3.21 \pm 1.45$ for fibrinogen) is a result of the different oxidation protocol. Albumin was oxidized for a much longer period and with higher ascorbate contents than fibrinogen (see Material and Methods).

\section{Effect of HCP on HD and HC Plasma}

Plasma from atherosclerotic patients on chronic HD treatment and from healthy subjects was evaluated before and after the application of HCP. The effect of HCP on inflammatory parameters and modified molecules in $\mathrm{HD}$ and HC plasma is stated in table 3. Significantly elevated levels of fibrinogen, CRP, IL- 6 and oxidized molecules such as advanced oxidative protein products, carbonylated fibrinogen and carbonylated albumin and significantly lower levels of albumin characterized the HD plasma. Following treatment by HCP, the levels of fibrinogen, albumin, CRP, TNF- $\alpha$, IL-6, AOPP, carbonylated fibrinogen, and carbonylated albumin significantly decreased in HD plasma. It must be emphasized that the pathologically high levels of fibrinogen and TNF- $\alpha$ in HD plasma were corrected to the normal healthy plasma level. In contrast, the only parameter that decreased significantly in $\mathrm{HC}$ treated by HCP was fibrinogen, which did not precipitate below the normal range (200-400 mg/ dl). In both groups, the levels of non-carbonylated albumin were hardly affected.

\section{Effect of HCP on the in vitro Precipitation of TNF- $\alpha$}

TNF- $\alpha$ is a main inflammatory cytokine involved in inflammatory states. Since high levels of TNF- $\alpha$ were found in HD plasma (table 3), we studied the effect of $\mathrm{HCP}$ on the non-traditional risk factor TNF- $\alpha$ precipitation in vitro. TNF- $\alpha$ concentrations before and after application of the HCP method were measured. HCP sig-

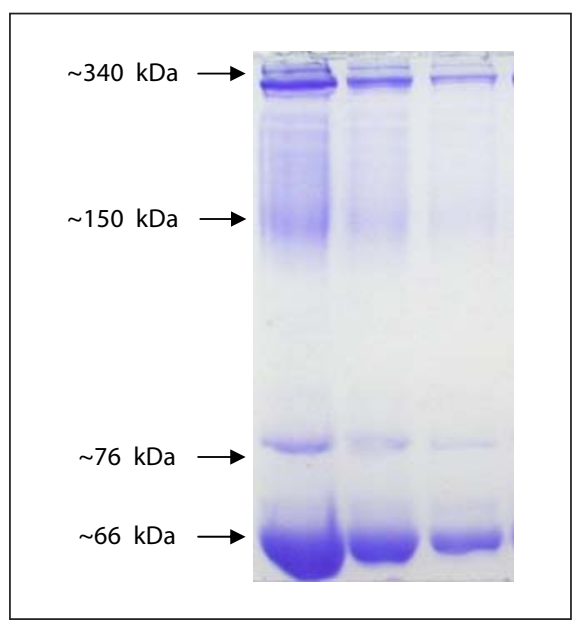

Fig. 3. Separation of HD cryogel on SDS-PAGE. A representative HD cryogel, loaded on to SDS-PAGE (8\%). The three lanes represent three different protein dilutions of the same HD cryogel.

nificantly precipitated commercial TNF- $\alpha$ levels from an average of $2.79 \pm 0.87$ to $2.03 \pm 0.45 \mathrm{pg} / \mathrm{ml}(-27 \%)$.

\section{Cryogel Composition}

SDS-PAGE Analysis of Cryogel. A representative picture of three different concentrations of HD cryogel as analyzed by SDS-PAGE is shown in figure 3. Cryogel was precipitated from HD plasma, separated on SDS-PAGE (8\%), stained by Coomassie blue and analyzed. The HD cryogel contained fibrinogen $(340 \mathrm{kDa})$, immunoglobulins $(150 \mathrm{kDa})$, transferrin $(76 \mathrm{kDa})$, and albumin $(66$ $\mathrm{kDa}$ ), in addition to other unidentified proteins (fig. 3).

Mass Spectrometry Analysis of Cryogel. The main proteins identified in the HD cryogel after analysis by mass spectrometry are depicted in table 4 . It can be seen that the cryogel contains fibrinogen, haptoglobin, immunoglobulins, transferrin, and albumin. This analysis is semiquantitative, and the detected proteins shown in table 4 are in a decreasing order as to their relative amounts in the cryogel. This analysis supported the results obtained by the SDS-PAGE (fig. 3).

Characterization of Fibrinogen/Carbonylated Fibrinogen in Plasma and Cryogel. Plasma and cryogel of HD and HC were separated on SDS-PAGE (6\%) and analyzed by Western blot analysis for fibrinogen and carbonylated fibrinogen (fig. 4a, b). HD plasma contained higher levels of fibrinogen, its degradation products, aggregates and carbonylated forms then form HC plasma. The HCP method reduced all forms of fibrinogen, which are abundant in the cryogel (fig. 4a, b). When comparing cryogel 


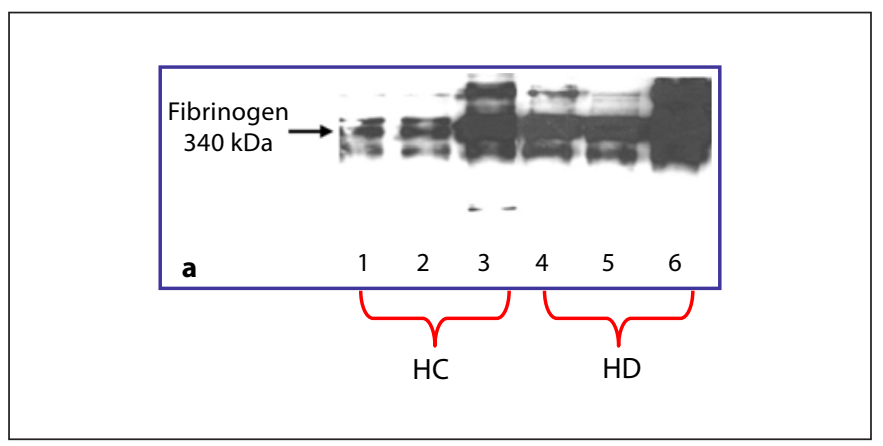

Fig. 4. Separation of plasma fibrinogen and carbonylated fibrinogen on SDS-PAGE. a Detection by anti-fibrinogen. Fibrinogen products from $\mathrm{HD} / \mathrm{HC}$ plasma and cryogel were separated on SDS-PAGE (6\%). The proteins were transferred to a nitrocellulose membrane. Western blot analysis was performed using rabbit polyclonal anti-fibrinogen serum and goat anti-rabbit-IgG peroxidase conjugate. Lanes 1-3 = HC, lanes 4-6 = HD; lanes 1 and 4 = before HCP, lanes 2 and $5=$ after HCP, lane $3=$ HC cryogel,

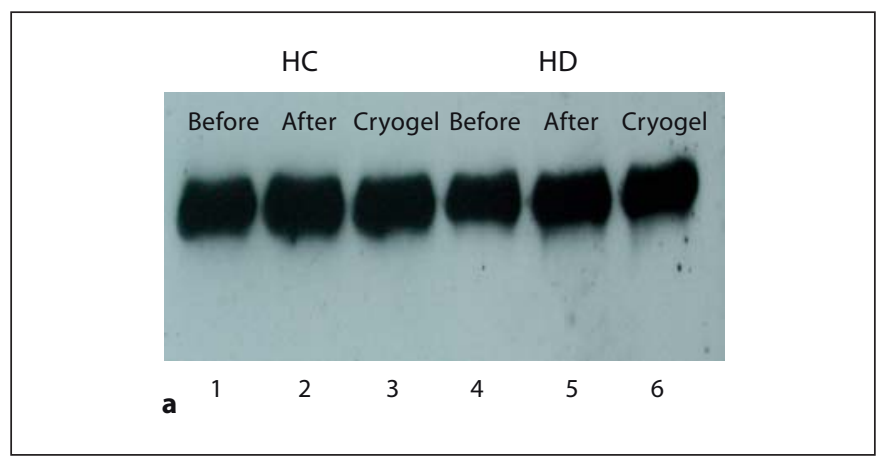

Fig. 5. Separation of plasma albumin and carbonylated albumin on SDS-PAGE. a Detection by anti-albumin. Albumin products from $\mathrm{HD} / \mathrm{HC}$ plasma and cryogel were separated on SDS-PAGE (10\%). The proteins were transferred to a nitrocellulose membrane. Western blot analysis was performed using rabbit polyclonal anti-albumin serum and goat anti-rabbit IgG peroxidase conjugate. Due to high albumin concentrations, plasma samples were diluted in loading buffer $1: 4$, and cryogel was diluted 1:2. Lanes 1-3 = HC; lanes 4-6 = HD; lanes 1 and $4=$ before HCP, lanes

from HC and HD it is evident that the amount of fibrinogen, its degradation products, aggregates and its carbonylated forms are precipitated to a greater extent in $\mathrm{HD}$ than in HC plasma (fig. 4a, b). The quantified fibrinogen and carbonylated fibrinogen levels in plasma are shown in table 3 and in cryogel in table 5 . It has to be noted that the oxidation causes higher molecular weight aggregates in HD than in HC (fig. 4b).

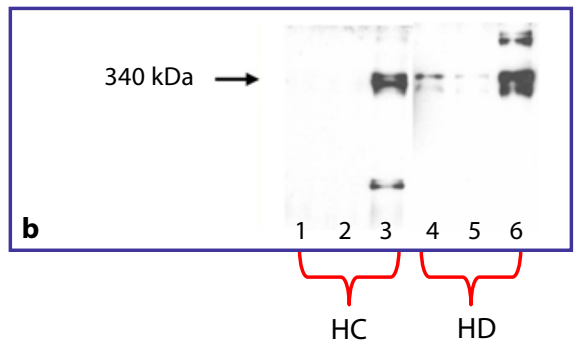

lane $6=\mathrm{HD}$ cryogel. b Detection with anti-DNP. Fibrinogen products from $\mathrm{HD} / \mathrm{HC}$ plasma and cryogel were derivatized with 2,4-dinitrophenylhydrazine, and separated on SDS-PAGE (6\%). The proteins were transferred to nitrocellulose membrane. For the detection of carbonyls, Western blot analysis was performed using rabbit polyclonal anti-DNP and goat anti-rabbit IgG peroxidase conjugate. The lanes are as specified in figure $4 \mathrm{a}$.

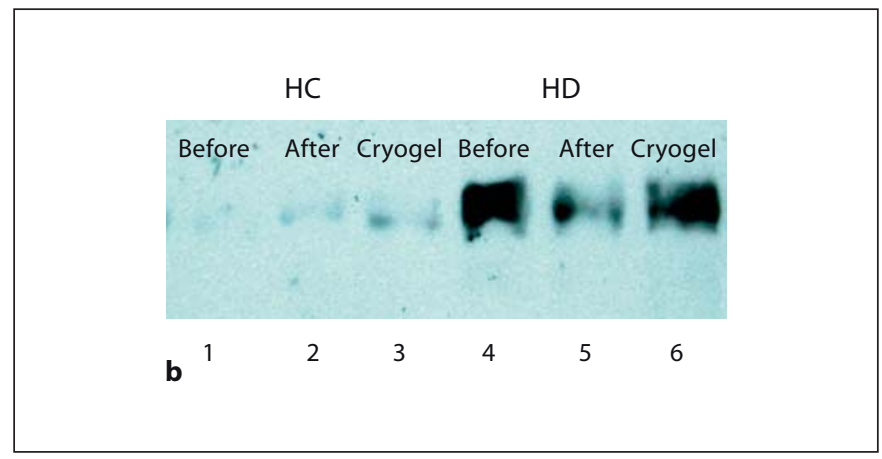

2 and $5=$ after HCP, lane $3=\mathrm{HC}$ cryogel, lane $6=\mathrm{HD}$ cryogel. b Detection with anti-DNP. Albumin from HD/HC plasma and cryogel was derivatized with 2,4-dinitrophenylhydrazine, and separated on SDS-PAGE (10\%). The proteins were transferred to nitrocellulose membrane. For the detection of carbonyls, Western blot analysis was performed using rabbit polyclonal anti-DNP and goat anti-rabbit IgG peroxidase conjugate. The lanes are as specified in figure $5 \mathrm{a}$.

Characterization of Albumin/Carbonylated Albumin in Plasma and Cryogel. Plasma and cryogel of HD and HC were separated on SDS-PAGE (10\%) and analyzed by Western blot analysis for albumin and carbonylated albumin (fig. 5a, b). HD plasma contained higher levels of carbonylated albumin than HC plasma, which was reduced by the HCP method (fig. 5b). Cryogel from HD showed significantly higher amounts of carbonylated albumin than HC cryogel (fig. 5b). The quantified levels of 
albumin and carbonylated albumin are shown in plasma (table 3) and in cryogel (table 5).

A summary of cryogel contents is depicted in table 5 . HD cryogel contained significantly higher levels of fibrinogen, CRP, TNF- $\alpha$, IL- 6 , AOPP, carbonylated fibrinogen and carbonylated albumin, while albumin levels were similar in both HD and HC cryogels.

\section{Discussion}

HCP, a unique molecular precipitating method, was developed in this study. This method combines the use of heparin, freezing temperatures and centrifugation to preferentially precipitate a cluster of risk factors from atherosclerotic patients' blood (in vitro).

During the development of the HCP method, we tried to find the optimal conditions to precipitate mainly modified molecules and cardiovascular risk factors while trying to avoid precipitation of native molecules. Preliminary studies evaluating the effect of various concentrations of heparin on precipitation level were carried out. These studies indicated that $14 \mathrm{IU} / \mathrm{ml}$ of sodium heparin is the lowest level of heparin required for application of the HCP method (data not shown). Heparin, a highly charged polyanion, is capable of forming soluble and insoluble complexes with proteins, which upon freezing and the application of centrifugal force will accelerate their precipitation $[17,18]$. The precipitate or the cryogel is insoluble in cold temperatures but mostly soluble at $37^{\circ} \mathrm{C}$, enabling its removal by centrifugation at lower temperatures. We show that heparin complexes of native proteins hardly precipitate, while complexes with these in vitro oxidized proteins (oxidized albumin and oxidized fibrinogen) precipitate better by freezing, thawing and centrifugation.

Plasma of atherosclerotic patients on chronic HD contains high levels of modified molecules and high levels of circulating non-traditional risk factors for atherosclerosis such as AOPP, carbonylated fibrinogen, carbonylated albumin, fibrinogen, CRP, IL- 6 and TNF- $\alpha$. All these parameters, together with the low levels of albumin, reflect a state of systemic oxidation and inflammation in these patients $[1-3,11,13,15,19,20]$.

When the HCP method was applied to plasma of HD patients, acute phase proteins and cytokines such as fibrinogen, CRP, IL-6, TNF- $\alpha$ and modified molecules such as AOPP, carbonylated fibrinogen and carbonylated albumin precipitated, while hardly any precipitation was observed in HC plasma. HD plasma contained signifi-
Table 4. Mass spectrometry analysis of the HD cryogel

\begin{tabular}{lc}
\hline Proteins & $\begin{array}{l}\text { Molecular weight } \\
\mathrm{kDa}\end{array}$ \\
\hline Albumin & 66 \\
Immunoglobulins & $140-900$ \\
Transferrin & 76 \\
Haptoglobin & $86-1,000$ \\
$\beta$-Globin & $\sim 16$ \\
Fibrinogen & 340 \\
Apoprotein A & $300-800$ \\
Ceruloplasmin & $\sim 130$ \\
Apoprotein C & $\sim 10$ \\
Von Willebrand factor & $>220$ \\
Complement components (C1q, C3, C4) & $190-410$ \\
\hline
\end{tabular}

Table 5. Cryogel contents: acute phase proteins, cytokines and modified molecules

\begin{tabular}{lcc}
\hline & HD cryogel & HC cryogel \\
\hline Fibrinogen, mg/dl & $239 \pm 114^{*}$ & $153 \pm 66$ \\
Albumin, g/dl & $0.38 \pm 0.14$ & $0.4 \pm 0.12$ \\
CRP, mg/l & $1.0 \pm 1.0^{*}$ & $0.1 \pm 0.1$ \\
TNF- $\alpha, \mathrm{pg} / \mathrm{ml}$ & $0.84 \pm 0.58$ & $0.78 \pm 0.33$ \\
IL-6, pg/ml & $0.96 \pm 0.67^{*}$ & $0.12 \pm 0.12$ \\
AOPP, $\mu \mathrm{M}$ & $26.6 \pm 10^{*}$ & $7.7 \pm 4.8$ \\
FIB-CO, nmol Carb/mg protein & $7.19 \pm 4.6^{*}$ & $<1.5$ \\
ALB-CO, nmol Carb/mg protein & $6.8 \pm 2.45^{*}$ & $2.37 \pm 1.23$ \\
\hline
\end{tabular}

Results are mean \pm SD of the following sample size: fibrinogen and albumin, $\mathrm{n}=15$ for HD, $\mathrm{n}=10$ for HC; CRP, TNF- $\alpha$, and IL- $6, \mathrm{n}=8$ for HD, $\mathrm{n}=4$ for HC; AOPP, FIB-CO and ALB-CO, $\mathrm{n}=10$ for $\mathrm{HD}, \mathrm{n}=5$ for HC. FIB-CO = Carbonylated fibrinogen; ALB-CO = carbonylated albumin.

${ }^{*} \mathrm{p}<0.05$ vs. HC cryogel.

cantly higher levels of AOPP, CRP, IL- 6 and TNF- $\alpha$ levels than $\mathrm{HC}$, which were significantly reduced by the HCP method.

It is interesting to note that in our healthy subjects, fibrinogen levels that were in the upper quartile of the normal range precipitated by the HCP to the lower normal levels. The risk of precipitating native molecules by HCP is one of the hazards of this method. Nevertheless, the described HCP method precipitated threefold more of the oxidized proteins compared to the native ones, with a minimal effect on the native ones. As other studies have shown that fibrinogen and its carbonylated form are in- 
flammatory mediators, we imply that significant reduction in their levels from HD plasma may be beneficial in attenuation of the atherosclerotic process and cardiovascular morbidities in these patients [3, 5, 13,21,22].

Hypoalbuminemia, frequently observed in HD patients, is a measure of malnutrition, acute phase inflammation, accelerated atherosclerosis (MIA syndrome), and a strong predictor of cardiovascular morbidity and mortality [23-26]. Himmelfarb and McMonagle [19] and Mera et al. [11] have reported that HD patients on chronic HD have lower albumin and higher carbonylated albumin levels than HC. We show that the HCP method is efficient in precipitating particularly the carbonylated albumin, without affecting the levels of the unmodified molecules both in HD patients and HC subjects. This is especially important in view of accumulating data showing that decreased albumin levels predict mortality in all populations, and are a powerful predictor of CVD in chronic HD patients [27]. Oxidized albumin, in addition to being a reliable marker for oxidative stress in HD patients [11], triggers an oxidative burst of human neutrophils and as such serves as a prooxidant too [9-11]. Thereby, the reduction in oxidized albumin levels from $\mathrm{HD}$ blood by HCP will contribute to the attenuation of the systemic oxidative stress in these atherosclerotic patients. A lack of interaction between the anionic albumin with the polyanionic heparin may explain the minor effect of heparin on the precipitation of the native albumin, nevertheless the HCP method precipitates better the oxidized form of albumin.

Our findings support the study by Himmelfarb and McMonagle [19] which demonstrated that albumin is the major plasma target of excess oxidation in HD patients. Albumin can accumulate oxidative modifications possibly due to its long turnover [28] in plasma (15-19 days); this, together with the high albumin concentration in plasma, compose albumin as the major plasma antioxidant protein.

AOPP is a surrogate marker of oxidative stress and serves as an indicator for protein oxidation [29]. AOPP also mediates inflammation and triggers oxidative reactions of neutrophils, monocytes and Tlymphocytes, which contribute to inflammation and to the impairment of the immune system found in atherosclerosis [30]. The reduction in AOPP levels by HCP from HD plasma, beyond confirming our hypothesis that HCP is an effective method in the reduction of modified molecules, may also be beneficial in reducing the inflammatory state in HD patients.

The reduction of CRP by HCP is also of biological importance. Beyond being a reliable marker for inflamma- tion and a strong predictor of future cardiovascular events [31], CRP is suggested as a mediator of the atherosclerotic process [32]. CRP production, which is predominantly under the control of IL- 6 , is involved in the induction of adhesion molecule expression in human endothelial cells, is able to activate the complement system [32], and may play a direct role in promoting the inflammatory component of atherosclerosis [31-33].

IL- 6 and TNF- $\alpha$ are central cytokines involved in the proinflammatory process. IL- 6 is suggested as a predictor of future heart disease and is the only cytokine that can stimulate the synthesis of all acute phase proteins involved in the inflammatory response [21].

TNF- $\alpha$ is regarded as the master regulator of the cytokine cascade. TNF- $\alpha$ induces thrombotic and inflammatory reactions in endothelial cells, and also induces production of IL- 6 by these cells [34]. We suggest that the highly significant reduction in TNF- $\alpha$ from HD plasma may result from its binding to heparin [35], forming a complex that is then precipitated by the HCP method.

By reducing the levels of all the mentioned cytokines and inflammatory mediators, a deceleration and retardation of the viscous cycle of oxidative-stress-inducing inflammation will be achieved, finally attenuating the atherosclerotic process.

As a mirror image, the HD cryogel contained significantly higher levels of fibrinogen, CRP, IL-6, AOPP, carbonylated fibrinogen, and carbonylated albumin compared to $\mathrm{HC}$ cryogel, confirming the enhanced precipitation of these inflammatory mediators and modified molecules from HD plasma.

Treatment of atherosclerosis is principally by the traditional pharmacological approach which deals mainly with one risk factor at a time, and is only partially successful in the reduction of CVD morbidity/mortality.

The heparin-mediated extracorporeal low-density lipoprotein precipitation procedure (HELP apheresis) is the main non-traditional technique used for selective removal of plasma low density lipoproteins in the indication of familial hypercholesterolemia unresponsive to medication. This procedure is based on application of high levels of heparin $(100 \mathrm{IU} / \mathrm{ml})$ together with the lowering of plasma $\mathrm{pH}$ values [36].

HELP apheresis manages to reduce levels of proatherogenetic lipoproteins and of several circulating proinflammatory markers as well [36]. Although HCP uses lower levels of heparin and does not require the lowering of the $\mathrm{pH}$ values, our results are in agreement with results presented by Wang et al. [37], showing reduction in fibrinogen and CRP levels. It has to be emphasized that 
HCP did not reduce fibrinogen levels below normal levels as in the data presented by Wang et al. [37].

Many atherosclerotic patients have normal levels of atherogenetic lipoproteins [38], suggesting that they are not always the major risk factor of atherosclerosis. These patients do not qualify for treatment by HELP apheresis.

In order to effectively attenuate the atherosclerotic process, there is a genuine need for a new therapeutic approach, such as HCP, which can simultaneously reduce multiple risk factors, independent of lipoprotein levels.

This study evaluated the effect of HCP on the in vitro precipitation of modified molecules and circulating nontraditional risk factors for atherosclerosis in plasma removed from HD patients. We believe that the data presented in this study may serve as a basis for future studies focusing on the development of an applicable in vivo clinical purification technique. Additionally, evaluation of the effect of HCP in vitro on plasma removed from other groups of atherosclerotic patients (such as patients with dyslipidemia/hypercholesterolemia) has to be carried out. The HCP method may be developed to complement the pharmacological treatments designed to reduce levels of circulating non-traditional risk factors for atherosclerosis and CVDs, in achieving attenuation in the propagation of atherosclerosis.

\section{Acknowledgments}

We are grateful to Prof. Vadim Krainess for his ideas and consultation during this study. We thank Mrs. Galina Shapiro for her assistance. We gratefully acknowledge the assistance of Dr. Michael Formanov in providing blood samples from HD atherosclerotic volunteers.

\section{References}

1 Muntner P, Hamm LL, Kusek JW, Chen J, et al: The prevalence of nontraditional risk factors for coronary heart disease in patients with chronic kidney disease. Ann Intern Med 2004;140:9-17.

-2 Descamps-Latscha B, Witko-Sarsat V, Nguyen-Khoa T, et al: Advanced oxidation protein products as risk factors for atherosclerosic cardiovascular events in nondiabetic predialysis patients. Am J Kidney Dis 2005;45:3947.

-3 Siato I, Folsom AR, Brancati FL, Duncan BB, et al: Nontraditional risk factors for coronary heart disease incidence among persons with diabetes: the Atherosclerosis Risk in Communities (ARIC) study. Ann Intern Med 2000;133:81-91.

4 Miyata T, Ueda Y, Yamada Y, et al: Accumulation of carbonyls accelerates the formation of pentosidine, an advanced glycation end product: carbonyl stress in uremia. J Am Soc Nephrol 1998;2349-2356.

-5 Shacter E: Quantification and significance of protein oxidation in biological samples. Drug Metab Rev 2000;32:307-326.

-6 Nystrom T: Role of oxidative carbonylation in protein quality control and senescence. EMBO J 2005;24:1311-1317.

7 Munch G, Berbaum K, Urban C, et al: Proteins of Thermus thermophilus are resistant to glycation-induced protein precipitation: an evolutionary adaptation to life at extreme temperatures? Ann NY Acad Sci 2005; 1043: 865-875.

8 Luthra M, Balasubramanian D: Nonenzymatic glycation alters protein structure and stability. A study of two eye lens crystallins. J Biol Chem 1993;268:18119-18127.
99 Faucette KJ, Fitzgerald LA, Liu L, Parker CJ, Rodgers GM: Characterization of the interactions between procoagulant albumin and human endothelial cells. Blood 1993;82: 2684-2692.

10 Anraku M, Yamasaki K, Maruyama T, et al: Effect of oxidative stress on the structure and function of human serum albumin. Pharm Res 2001;18:632-639.

11 Mera K, Anraku M, Kitamura K, et al: The structure and function of oxidized albumin in hemodialysis patients: its role in elevated oxidative stress via neutrophil burst. Biochem Biophys Res Commun 2005;334:13321328.

12 Shacter E, Williams JA, Lim M, et al: Differential susceptibility of plasma proteins to oxidative modification: examination by Western blot immunoassay. Free Radic Biol Med 1994;17:429-437.

$\checkmark 13$ Azizova OA, Maksyanina EV, Romanov YA, Aseichev AV, Scheglovitova ON: Fibrinogen and its oxidized form induce interleukin- 8 production in cultured endothelial cells in human vessels. Bull Exp Biol Med 2004;137: 358-360.

14 Smith JW, Kayashima K, Katsume C, et al: Cryopheresis: immunochemical modulation and clinical response in autoimmune disease. Trans Am Soc Artif Intern Organs 1982;28:391-395.

15 Michelis R, Gery R, Sela S, et al: Carbonyl stress induced by intravenous iron during haemodialysis. Nephrol Dial Transplant 2003; 18:924-930.
16 Witko-Sarsat V, Friedlander M, CapeillereBlandin C, et al: Advanced oxidation protein products as a novel marker of oxidative stress in uremia. Kidney Int 1996;49:1304-1313.

17 Siami GA, Siami FS: Current topics on cryofiltration technologies. Ther Apher 2001;5: 283-286.

18 Nose Y, Horiuchi T, Malchesky PS, et al: Therapeutic cryogel removal in autoimmune disease: What is Cryogel? Ther Apher 2000; 4:38-43.

19 Himmelfarb J, McMonagle E: Albumin is the major plasma protein target of oxidative stress in uremia. Kidney Int 2001;60:358363.

20 Stenvinkel P, Ketteler M, Jonson RJ, et al: IL10 , IL- 6 , and TNF- $\alpha$ : central factors in the altered cytokine network of uremia - the good, the bad and the ugly. Kidney Int 2005; 67:1216-1233.

21 Woods A, Brull DJ, Humphries SE, et al: Genetics of inflammation and risk of coronary artery disease: the central role of interleukin-6. Eur Heart J 2000;21:1574-1583.

-22 Duperray A, Languino LR, Plescia J, et al: Molecular Identification of a novel fibrinogen binding site on the first domain of ICAM-1-1 regulating leukocyte-endothelium bridging. J Biol Chem 1997;272:435441.

23 Mitch WE: Malnutrition: a frequent misdiagnosis for hemodialysis patients. J Clin Invest 2002;110:437-439.

24 Beddhu S, Kaysen GA, Yan G, Sarnak M, Agodoa L, Ornt D, Cheung AK; HEMO Study Group: Association of serum albumin and atherosclerosis in chronic hemodialysis patients. Am Kidney Dis 2002;40:721-727. 
25 Oh DJ, Lee KJ: The relation between hypoalbuminemia and compliance and intima-media thickness of carotid artery in continuous ambulatory peritoneal dialysis patients. J Korean Med Sci 2005;20:70-74.

26 Danielski M, Ikizler TA, McMonagle E, et al: Linkage of hypoalbuminemia, inflammation and oxidative stress in patients receiving maintenance hemodialysis therapy. Am J Kidney Dis 2003;42:286-294.

$\checkmark 27$ Kaysen A, Don BR: Factors that affect albumin concentration in dialysis patients and their relationship to vascular disease. Kidney Int 2003;63:94-97.

-28 Volwiler W, Goldsworthy PD, MacMartin $\mathrm{M}$, et al: Biosynthetic determination with radioactive sulfur of turnover rates of various plasma proteins in normal and cirrhotic man. J Clin Invest 1955;34:1126-1146.

29 Witko-Sarsat V, Friedlander M, Nguyen TN, et al: Advanced oxidation protein products as novel mediators of inflammation and monocyte activation in chronic renal failure. Immunology 1998;161:2524-2532.
30 Skvarilova M, Bulava A, Stejskal D, et al: Increased levels of advanced oxidation products as a marker of oxidative stress in patients with acute coronary syndrome. Biomed Papers 2005; 149:83-87.

31 Jialal I, Devaraj S, Venugopal SK: C-reactive protein: risk marker or mediator in atherothrombosis. Hypertension 2004;44:6-11.

$\checkmark 32$ Otto C, Geiss HC, Empen K, et al: Long-term reduction of $\mathrm{C}$-reactive protein concentration by regular LDL apheresis. Atherosclerosis 2004; 174:151-156.

33 Agrawal A: CRP after 2004. Mol Immunol 2005;42:927-930.

34 Yamagishi SI, Inagaki Y, Nakamura K, et al: Pigment epithelium-derived factor inhibits TNF- $\alpha$ induced interleukin- 6 expression in endothelial cells by suppressing NADPH oxidase-mediated reactive oxygen species generation. J Mol Cell Cardiol 2004;37:497506.
35 Harvima TI, Lappalainen K, Hirvonen MR, et al: Heparin modulates the growth and adherence and augments the growth-inhibitory action of TNF- $\alpha$ on cultured human keratinocytes. J Cell Biochem 2004;92:372386.

36 Mellwig KP: Heparin-induced extracorporeal low density lipoprotein precipitation. Ther Apher Dial 2003;7:365-369.

37 Wang Y, Blessing F, Walli AK, et al: Effect of heparin-induced extracorporeal low density lipoprotein precipitation beyond lowering proatherogenetic lipoproteins - reduction of circulating proinflammatory and procoagulatory markers. Atherosclerosis 2004; 175 : 145-150.

38 Bolton CH, Downs LG, Jason GG, et al: Endothelial dysfunction in chronic renal failure: role of lipoprotein oxidation and proinflammatory cytokines. Nephrol Dial Transplant 2001;16:1189-1197. 